\title{
Research on battery capacity reliability of intelligent power supply equipment
}

\author{
Zikuo Dai ${ }^{1, *}$, Yan $\mathrm{Xu}^{2}$, Kejian $\mathrm{Shi}^{3}$, Yang $\mathrm{You}^{4}$ and Bingxin $\mathrm{Li}^{4}$ \\ ${ }^{1}$ State Grid Liaoning Electric Power Company, Shenyang, Liaoning 110000, China \\ ${ }^{2}$ Tieling Power Supply Company of State Grid Liaoning Electric Power Co. LTD., Tieling, Liaoning 112000, China \\ ${ }^{3}$ State Grid Liaoning Electric Power Research Institute, Shenyang, Liaoning 110000, China \\ ${ }^{4}$ Panjin Power Supply Company of State Grid Liaoning Electric Power Co. LTD., Panjin, Liaoning 124010, China
}

\begin{abstract}
Battery is not only the power source or emergency power supply for many ships, but also the essential emergency power supply for train command and dispatching, post and telecommunication, bank operation, etc. The capacity of battery will be directly related to the normal operation of ship train dispatching, post and telecommunication and bank operation. At the same time, the battery is also the last guarantee for the safe and reliable power supply of intelligent power supply equipment, so it is necessary to study the reliability of battery capacity
\end{abstract}

\section{Introduction}

Most of the batteries used in modern intelligent power supply equipment are VRLA batteries. The voltage of the battery terminal in the floating state can not fully reflect the capacity of the battery. Even if the capacity of the battery becomes smaller, the qualified terminal voltage can be measured in the floating charge state, so the check discharge can only be carried out offline. The common detection methods of battery capacity are to measure the terminal voltage of single battery and discharge the battery capacity once a year. Usually, the battery is working in the floating charge state. During the discharge period and the recharging period after the discharge, the battery can not guarantee the discharge requirements under the power-off state, which leads to artificial communication interruption. Therefore, the realization of on-line detection of DC system battery capacity is of great significance to improve the safe operation of DC system and the reliability and automation of DC power supply. This paper mainly focuses on the battery capacity of intelligent power supply equipment, so as to ensure the safety and efficiency of the whole intelligent power supply equipment.

\section{The basic characteristics of battery in intelligent power supply equipment}

Nowadays, lead-acid batteries are almost used in intelligent power supply equipment, so it is necessary to study lead-acid batteries.

\subsection{Density characteristics}

The positive and negative electrodes of lead-acid batteries are mainly composed of lead, and the electrolyte is sulfuric acid, so they are also called acid batteries. The charge and discharge of lead-acid battery depend on the reversible reaction of chemical substances in the battery. During the discharge, the sponge like lead on the negative plate and the lead dioxide on the positive plate are gradually transformed into lead sulfate. At the same time, a part of sulfuric acid is consumed, and a part of water is added. The concentration of electrolyte decreases and the density decreases. When charging, with the passage of current, the lead sulfate on the negative plate changes into dark gray sponge like lead, and the lead sulfate on the positive plate changes into dark brown lead dioxide. Sulfuric acid is generated in the solution, and the composition of sulfuric acid in the electrolyte increases, the moisture decreases, the electrolyte concentration increases, and the density increases. Therefore, the method of measuring density can be used to judge the degree of charge and discharge of the battery ${ }^{[1]}$.

\subsection{Voltage characteristics}

The potential of lead-acid battery is about $2.1 \sim 22 \mathrm{~V}$ after charging and about ZV when discharging. During charging, the potential increases to $2.5 \sim 26 \mathrm{~V}$. If charging is continued, because the effective substances on the plate have been reduced completely ${ }^{[2]}$, the decomposition of water tends to be saturated gradually. At this time, the electrolyte only boils, but the voltage is stable at about $2.7 \mathrm{~V}$ and will not increase. After stopping

\footnotetext{
" Corresponding author: E-mail: daizikuo1985@163.com
} 
charging, the potential of the battery will drop sharply to $2.3 \mathrm{~V}$, and finally stabilize at about $2.1-2.2 \mathrm{~V}$. When discharging, the potential drops to $2-2.05 \mathrm{~V}$. Then, for a long time, the potential drops slowly and steadily. When the discharge reaches a certain voltage (i.e. termination voltage), the potential of the battery begins to drop sharply, which is the symbol of the end of discharge. However, due to the phenomenon of "rest" when the battery discharges, the potential of the open circuit battery can not indicate the degree of discharge.

\subsection{Temperature characteristic}

The heating factors of lead-acid batteries are as follows:

(1) Internal resistance heat. There is a certain internal resistance in lead-acid battery, which generates internal resistance heat through current.

(2) Reversible heat. The lead-acid battery has such properties: when discharging, the potential will drop, and some of it will be absorbed and turned into electric energy output, so it will cause the temperature of electrolyte to drop; when charging, the potential will rise, and some of it will be consumed reversibly, and the same heat will be released, so that the temperature of electrolyte will rise.

(3) Heat of dilution. Sulfuric acid and water are mixed to dilute sulfuric acid and give off heat. When the battery is charging, the sulfuric acid formed in the pores diffuses and dilutes outside the pores, and the dilution heat causes the electrolyte temperature to rise.

(4) After charging. When electric energy is used to electrolyze water, part of the heat will be generated to raise the electrolyte temperature ${ }^{[3]}$. The normal operation temperature of lead-acid battery is $20 \sim 50{ }^{\circ} \mathrm{C}$, too high temperature will have a very adverse impact on the capacity and life of the battery. The best operating temperature is $25^{\circ} \mathrm{C}$. When the temperature increases by $5{ }^{\circ} \mathrm{C}$, the service life of the battery will be reduced by $10 \%$.

\subsection{Liquid level characteristics}

In the process of charging and discharging, sulfuric acid and water participate in the reaction, which will inevitably lead to the increase and decrease of electrolyte level. The electrolyte level of marine lead-acid battery should be higher than 30nun $\sim 75 \mathrm{innl}$, but not beyond this range ${ }^{[4]}$.

\section{Design of common detection methods for battery capacity of intelligent power supply equipment}

\subsection{Calculation of battery impedance of intelligent power supply equipment}

In the early 1960s, ErshIeret and RandIes proposed the method of collecting and measuring electrode impedance as a frequency function to study battery capacity ${ }^{[5]}$. In recent years, many people have popularized this method, and it has been applied in many aspects, such as lowfrequency impedance extrapolation method, which can easily measure the change of battery impedance during charging and discharging.

Supposing that the two electrodes of the battery keep a constant potential ${ }^{[6]}$, and a direct current flows between them. A small amplitude sinusoidal alternating voltage is applied at both ends of the battery to make the current in the battery contain a small amplitude sinusoidal alternating current. The equivalent circuit of battery electrical parameters is shown in Figure 1.

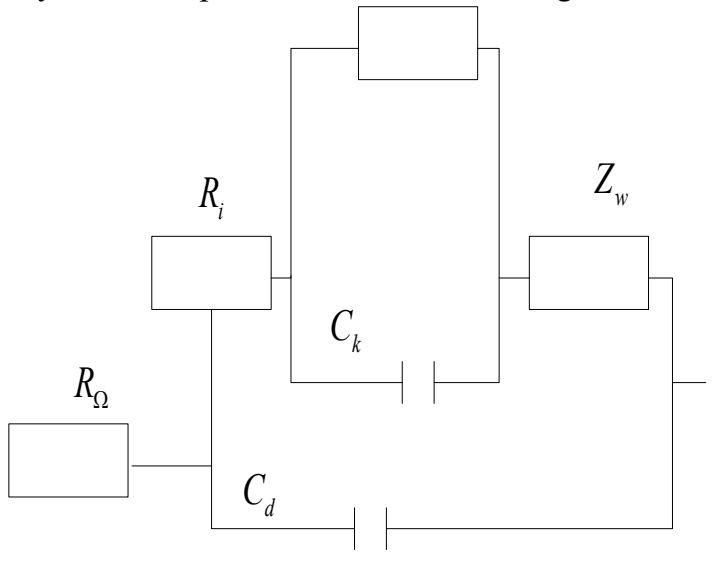

Fig.1 Battery equivalent circuit analysis diagram

In Figure $1, Z_{w}$ auxiliary is connected in parallel with $R_{k}$ and $R_{\Omega}, R_{i}$ is AC complex plane impedance, which is called Waburg impedance. It is caused by concentration polarization of electrode, and it is related to capacitive reactance and resistance characteristics of electrode reaction. The parallel connection of $R_{k}$ indicates the constant time of battery electromotive force [7]. The AC current refers to the lead AC voltage $u, C_{k}$ as the internal resistance of electrode reaction, which indicates the characteristic that the $\mathrm{AC}$ current distributed on the electrode surface can not change immediately when the AC voltage changes. $C_{d}$ is an electrode double layer capacitor. In the low-frequency measurement process, because the concentration polarization and electric double-layer capacitance of the battery can be ignored, so / $\mathrm{w} \rightarrow 0$, the impedance $z$ equation of the battery is usually written in complex form:

$$
\begin{gathered}
Z=\left(R_{\Omega}+R_{\mathrm{t}}\right)+\frac{1}{\frac{1}{R_{\mathrm{k}}}+\mathrm{j} \omega C_{\mathrm{k}}} \\
=\left(R_{\Omega}+R_{\mathrm{t}}\right)+\frac{R_{\mathrm{k}}}{1+\left(\omega C_{\mathrm{k}} R_{\mathrm{k}}\right)^{2}}-\mathrm{j} \frac{R_{\mathrm{k}}\left(\omega C_{\mathrm{k}} R_{\mathrm{k}}\right)}{1+\left(\omega C_{\mathrm{k}} R_{\mathrm{k}}\right)^{2}}
\end{gathered}
$$

The AC impedance curve of storage battery consists of the following four parts: 1 . The inductive part when the frequency is lower than $100 \mathrm{~Hz} ; 2$. The real part of high frequency resistance $\mathrm{RH}$ after exceeding $100 \mathrm{~Hz}^{[8]}$; 
3. The first small capacitive ring between 0.1 and $100 \mathrm{~Hz}$ (radius $\mathrm{r} 1$ ); 4. The second capacitive ring below $0.1 \mathrm{~Hz}$ (radius r2). The general interpretation of battery impedance spectrum is as follows: 1 . The inductive part of the part over $100 \mathrm{~Hz}$ is affected by the internal geometry and connecting parts of the battery; 2. OHMIC resistance RHF includes connecting resistance, diaphragm resistance, electrolyte resistance and lead sulfate crystal junction resistance; 3 . The small capacitive ring is related to the porosity of the electrode; 4. The large capacitive ring relies on the electrode reaction, The rate is limited by the $p$ mass transfer rate. Therefore, the impedance of the battery is a complex impedance, which is related to the test frequency when other conditions remain unchanged. The internal resistance of battery is the mode of complex impedance, so the internal resistance of battery also refers to the internal resistance value at a certain test frequency. In this way, according to the above calculation, we can get the battery impedance in combination with the spectrum [15].

\subsection{Analysis of the relationship between energy stored in capacitor and capacitance value}

The energy stored in the flexible battery terminal capacitor has the following relationship with its capacitance value and terminal voltage:

$$
E_{s c}=\frac{1}{2} \cdot C \cdot U_{s c}^{2}
$$

In formula (2), $C$ represents the capacitance value of capacitor energy storage, $E_{s c}$ represents the capacitance value of capacitor energy storage, and $U_{s c}^{2}$ represents the voltage value of capacitor terminal.

The energy analysis strategy of this study is to implement quota analysis in energy management. First, start the smoothing analysis, then the management analysis. Through the smoothing analysis, the capacitor of the battery pack and the charging and discharging power target of the battery can be adjusted, but the smoothing control cannot adjust the output result of the smoothing control. The correction values of the output active power of battery and super-capacitor are calculated by limit management strategy

$$
\Delta R_{\text {bat }}=Q / \Delta P_{s c}
$$

In formula (3), $\Delta R_{b a t}$ represents the battery active power correction, $\Delta P_{s c}$ represents the battery capacitor output power, and $Q$ represents the active power deficiency.

It can be seen from the above formula that the battery energy storage is the backup energy storage of the super capacitor. When the super capacitor capacity reaches the upper (lower) limit, the battery is responsible for compensating the active power shortage of the super capacitor. Based on the above process, the capacitor voltage of the flexible battery is pre analyzed ${ }^{[19]}$.

\subsection{Battery capacity and internal resistance measurement}

This is the "half load measurement method" which is between the two and has the advantages of both. The following data can be obtained by using "half load measurement method":

For the convenience of discussion, ignoring the difference of different internal resistance measuring instruments, the same method of drawing voltage curve family can also be used to draw the internal resistance curve family of battery under discharge. The variation law of internal resistance under discharge state is not as familiar as that of voltage, but it has the following characteristics after a lot of research,

1) There is little change above $50 \%$ charge rate.

2) Rapid rise below $50 \%$ charge rate.

3) Before the end of discharge, the internal resistance value may rise to 2-4 times of the initial internal resistance value.

4) The $50 \%$ charge rate is the inflection point of the internal resistance curve, which can be roughly represented by the two fold line method.

In order to clearly express the change law of internal resistance curve family, a representative battery model is selected. The model group is composed of three batteries with nominal capacity of $1000 \mathrm{~A} \cdot \mathrm{h}$, and the actual capacity of $1000 \mathrm{~A} \cdot \mathrm{h}, 800 \mathrm{~A} \cdot \mathrm{h}$ and $600 \mathrm{~A} \cdot \mathrm{h}$ represent three typical types of battery group, namely good, medium and bad. The internal resistance of floating charge is $0.20 \mathrm{~m} \Omega$, $0.20 \mathrm{~m} \Omega$ and $0.27 \mathrm{~m} \Omega$ respectively. Please note that the internal resistance of $1000 \mathrm{~A} \cdot \mathrm{h}$ and $800 \mathrm{~A} \cdot \mathrm{h}$ is equal to $0.20 \mathrm{~m} \Omega$, which is supported by the measured data. It also indicates that the internal resistance distribution under full power does not conform to the correlation law of "large internal resistance and small capacity". It is assumed that the internal resistance at the end of discharge is three times of the initial internal resistance.

The terminal point is 3 times of $0 \%$ true charge rate and initial internal resistance, and the inflection point is the slightly larger value of $50 \%$ true charge rate and initial internal resistance.

The above is the battery capacity and internal resistance measurement used in the half load measurement method can get all the measurement data.

\section{Experiment}

The battery pack of an intelligent power supply equipment is made of $242 \mathrm{~V} / 20(\mathrm{~mA})$ batteries in series. The internal resistance of the battery has been tested and the battery capacity has been converted by using the experimental device in this paper. The standard instrument used in the experiment is the BcT-2 + 0 battery capacity test system produced by njber company of the United States, and the measurement accuracy is \pm 0.5 .

The experiment mainly compares the traditional method with the method of this study, and compares the measurement error and measurement time of the two methods. 


\subsection{Comparison of measurement error}

The measurement error between the measurement method in this study and the traditional method is as follows:

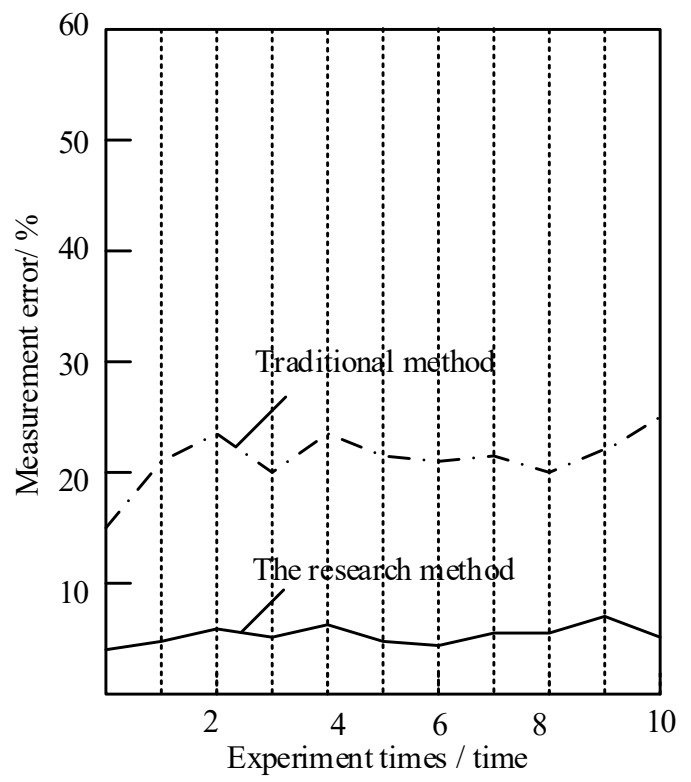

Fig.2 Comparison of measurement error

Through the analysis of Figure 2, it can be seen that the measurement error of the analysis method in this study is less than that of the traditional method.

\subsection{Measurement time comparison}

The results are as follows:

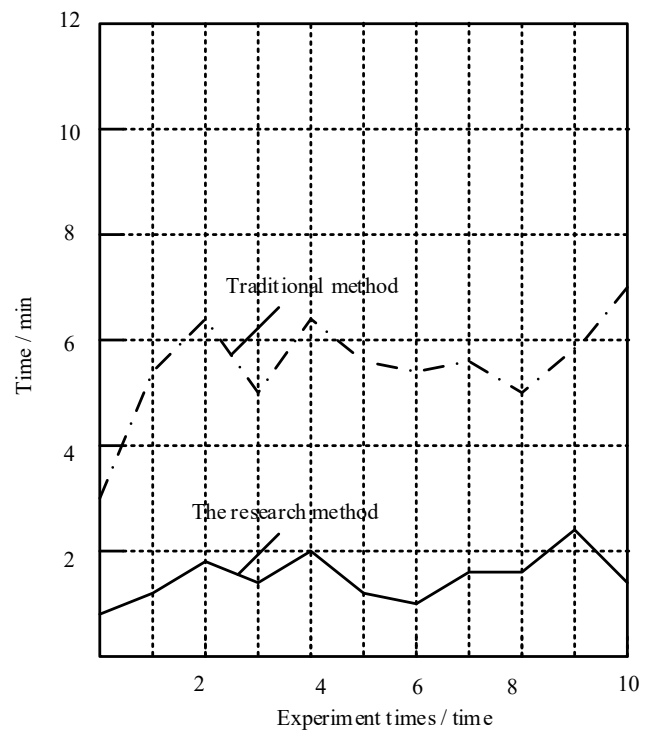

Fig.3 Measurement time comparison

It can be seen from the above figure 3 that the measurement time of this study is less than that of traditional methods, and the application effect is better than that of traditional methods.

Because the storage battery usually works in the floating charge state, most of the time it works in the full capacity or near full capacity state, the test method proposed in this paper solves this problem well.

\section{Conclusion}

This paper presents the research on the reliability of battery capacity of intelligent power supply equipment. This research ensures the real-time online detection of battery capacity and internal resistance, and optimizes the correlation between battery internal resistance and capacity. This paper provides a reliable basis for accurately determining the residual capacity of the battery.

\section{Reference}

1. Nara, H.,Yokoshima, T., Mikuriya, H., et al.(2017) The Potential for the Creation of a High Areal Capacity Lithium-Sulfur Battery Using a Metal Foam Current Collector. J. Electrochemical Society, 164:5026-5030.

2. Baek, M. K., Park, J. B., Son, S. Y., et al.(2018) A Study of Optimum Capacity of Battery Energy Storage System Linked PV. Transactions of the Korean Institute of Electrical Engineers, 67:38-45.

3. Li, Y., Khurram, A., Gallant, B. M.(2018) A HighCapacity Lithium-Gas Battery Based on Sulfur Fluoride Conversion. J. Physical Chemistry C, 122:7128-7138.

4. Qian, C., Zhao, J., Sun, Y., et al.(2020) ElectrolytePhobic Surface for the Next-Generation Nanostructured Battery Electrodes. Nano Letters, 20:7455-7462.

5. Alam, M. J. E., Muttaqi, K. M., \&Sutanto, D.. (2017) Effective utilization of available pev battery capacity for mitigation of solar pv impact and grid support with integrated $v 2 g$ functionality. IEEE Transactions on Smart Grid,7: 1562-1571.

6. Kwon, Y. H., Minnici, K., Park, J. J., Lee, S. R., Zhang, G., \& Takeuchi, E. S., et al. (2018) Swnt anchored with carboxylatedpolythiophene "links" on high-capacity li-ion battery anode materials. Journal of the American Chemical Society,140: 5666-5669.

7. Kenichi, Ogawa, Keiichiro, \& Kondo. (2019) Design method of installed fuel cell power and lithium-ion battery capacity for fuel cell hybrid railway vehicles. IEEJ Transactions on Industry Applications, 139: 472-479.

8. Gao, Z., Lin, Z., Laclair, T. J., et al. (2017) Battery capacity and recharging needs for electric buses in city transit service. Energy,122: 588-600. 